\title{
Constructions détachées en rupture, comportant une forme verbale non finie : éléments de comparaison entre français classique du début du XVIIle siècle et français contemporain
}

\author{
Nathalie Rossi-Gensane \\ CLLE ERSS (UMR 5263), Université de Toulouse, Site de Toulouse 2 \\ nrossi@univ-tlse2.fr
}

\section{Introduction*}

Après avoir défini les constructions détachées comportant une forme verbale non finie, on se restreindra à celles, dites en rupture, dont le «référent sous-jacent» ne correspond pas au sujet du prédicat premier. L'objet de cette étude est de comparer, dans une perspective avant tout syntaxique, quelques tendances de l'usage (littéraire) classique du premier tiers du XVIIIe siècle, d'une part, et de l'usage contemporain, d'autre part, dans le cadre de ce qui pourrait être assimilé à un mouvement de grammaticalisation, la structure phrastique prenant graduellement le pas sur l'organisation discursive. On décrira certaines sortes de ruptures communes au français classique et au français contemporain, qui affectent dans une grande mesure les constructions infinitives et gérondives. Les différences portent principalement sur les constructions détachées thématisées, représentées notamment par des formes participiales en -ant. On s'attachera à caractériser les contextes d'apparition de ces ruptures spécifiques, et à montrer de quelle façon, si l'on rapproche ces dernières de certaines ruptures en français contemporain, ces contextes sont devenus beaucoup plus contraints à cause de l'affaiblissement du rôle de continuité référentielle de la construction détachée.

\section{Présentation de quelques notions}

\subsection{Les constructions détachées comportant une forme verbale non finie}

Combettes (1998, 4ème de couverture) définit les constructions détachées comme :

\begin{abstract}
une série d'expressions, différentes d'un point de vue formel (adjectifs, participes, [syntagmes] introduits par une préposition, constructions absolues), mais qui présentent des propriétés communes dont les principales sont : la liberté de position dans la phrase [...], la valeur d'un prédicat secondaire qui vient s'ajouter à la prédication principale, la présence d'un référent sous-jacent auquel la construction détachée doit renvoyer.
\end{abstract}

On rappellera que sont rangés dans les formes verbales non finies le participe (présent et passé), le gérondif et l'infinitif, unités non chronologiques et non personnelles. Ont donc été intégrés dans les constructions détachées à forme verbale non finie les participes, présents et passés, détachés, les gérondifs, ainsi que (conformément à Charolles et Combettes, 1999, mais à la différence de Combettes, 1998) certains infinitifs prépositionnels. Le participe, présent ou passé, «forme adjectivale » (cf. Fournier, 1998, p. 304), exerce, détaché, la fonction d'apposition ${ }^{1}$. Le gérondif, qui participe de l'adverbe, est complément circonstanciel, comme l'est l'infinitif, quant à lui mode nominal du verbe, lorsqu'il est construction détachée. Signalons que les gérondifs et les infinitifs prépositionnels circonstants ne sont pas toujours précédés d'une virgule, l'absence de cette dernière ne préjugeant pas nécessairement du caractère intégré ou détaché du syntagme ${ }^{2}$. 


\title{
2.2 La notion de contrôleur
}

A la notion de «référent sous-jacent", d'ordre sémantico-référentiel, on préférera celle de contrôleur, issue de la grammaire générative ${ }^{3}$ mais utilisée par la suite dans un sens large par exemple par Le Goffic (1993) et Fournier (1998). Ainsi, dans Pierre craint de parler, le contrôleur de l'infinitif parler, encore appelé « sujet virtuel» (Le Goffic, 1993, p. 35), ou « sujet sémantique » (Fournier, 1998, p. 293), est Pierre.

\subsection{Les constructions en rupture ou le non-respect de la règle de coréférence}

Pour la grammaire normative, le contrôleur de la construction détachée doit être le sujet du prédicat (premier). On citera, parmi de nombreux autres ouvrages, Grevisse et Goosse (1988) :

\begin{abstract}
Pour la clarté de l'expression, il est souhaitable que l'épithète (notamment le participe, auquel on peut joindre le gérondif) placée au début de la phrase (ou de la proposition) se rapporte au sujet de cette phrase (ou de cette proposition). (p. 548)

Pour la clarté, il est souhaitable que l'agent de l'infinitif soit identique au sujet du verbe support. (p. 1336)
\end{abstract}

tout en soulignant que la règle, ancienne, est notamment prônée par Bouhours ${ }^{4}$, qui caractérise les constructions non conformes comme étant «en l'air », Régnier-Desmarais ${ }^{5}$ et l'Académie Française ${ }^{6}$.

A travers quelques romans du premier tiers du XVIIIe siècle ${ }^{7}$, on se demandera quelles spécificités de l'usage classique, par rapport à la norme moderne, mais aussi et surtout par rapport à l'usage contemporain, peuvent être dégagées. En effet, Fournier (1998) signale, aussi bien pour l'infinitif complément circonstanciel, le gérondif et le participe détaché, présent ou passé, que l'usage classique se caractérise par la liberté de repérage du contrôleur, mais, également, que cet usage classique, s'il est éloigné de la norme moderne, est proche de l'usage moderne réel.

\subsection{L'identification des gérondifs parmi les participes apparents}

Au premier abord, les constructions détachées en rupture à forme verbale non finie comportent de nombreuses formes participiales en -ant. Cependant, on sait qu'en français classique, les gérondifs ne sont pas régulièrement marqués par $e^{8}$. Fournier (1998, p. 293), à la suite de Régnier-Desmarais, propose le critère de la paraphrase possible par en $\mathrm{V}$-ant : si l'apparent participe présent est un gérondif, il peut être précédé de en sans véritable changement de sens. Or, dans les œuvres étudiées, la plupart des occurrences ne permettent pas cette paraphrase, ce qui confirme la constatation de Halmøy (2003, p. 56) :

\footnotetext{
Si les grammairiens du XVIIe siècle continuent à souligner les difficultés que l'on a à départager gérondifs et participes présents dans la langue classique, un petit sondage dans la correspondance de Madame de Sévigné [dans le dernier quart du XVIIe siècle] montre que l'emploi des gérondifs correspond là déjà tout à fait à son emploi moderne : ils y sont nombreux [...].
}

Dans certains cas, le remplacement par la séquence en $\mathrm{V}$-ant est possible mais induit, à tort, une interprétation de coréférence avec le sujet, cf. De-là vient que nous n'avons d'eclipse de lune que lorsque se levant d'un côté, pendant que le soleil se couche de l'autre, et que ces deux astres sont par conséquent en oposition, la terre se trouve directement entre-deux, et empêche qu'ils ne se puissent voir en face. (Tyssot, p. 96) $)^{9}$ Dans la plupart des cas, l'alternance est impossible, parfois en raison de la contrainte de dynamicité. Selon Halmøy (2003), cette contrainte empêche le gérondif - sauf s'il exprime un repère temporel ou une relation de concomitance - d'apparaitre dans une configuration statique, c'est-à-dire de porter sur un verbe recteur statique, ou d'être lui-même doté d'une valeur aspectuelle d'état ${ }^{10}$, cf. il $\underline{m e}$ seroit fâcheux que n'étant pour rien dans vos extravagances, on me crût capable de les partager (Crébillon, p. 358), où le verbe non fini, être, a une valeur éminemment statique. L'alternance est également presque toujours exclue lorsque la forme en -ant est composée et aboutit à un participe passé, peut-être en relation avec la contrainte qui vient d'être citée, dans la mesure où la forme verbale non finie, 

apparement plus large que le nôtre, se trouva tout d'un coup embarrassé [...]. (Tyssot, p. 60)

\subsection{Ruptures totales vs ruptures partielles}

On peut au premier abord être tenté de distinguer entre ruptures partielles, où le contrôleur apparaît dans la phrase mais n'est pas sujet (du prédicat premier), et ruptures totales, où le contrôleur n'apparaît pas dans la phrase. Les ruptures semblent alors, dans leur immense majorité, partielles, l'occurrence suivante - d'où est absent le contrôleur, je - constituant une exception : Déchirée par ces deux mouvemens, ils ne cessoient qu'à votre vue [...]. (Crébillon, p. 579)

Dans un deuxième temps, on peut se demander si, pour de nombreuses occurrences, une autre analyse n'est pas possible, c'est-à-dire si le contrôleur apparaît véritablement dans le cadre de la phrase, ou bien s'il n'est pas parfois un élément du contexte gauche, hors du cadre phrastique, repris dans ce cadre par un terme en diverses fonctions. Ainsi, dans L'ayant fait capable de discernement, il étoit bien juste qu'il vécût sous sa dépendance [...] (Tyssot, p. 165), le contrôleur est-il le déterminant possessif sa, repéré dans le cadre de la phrase, ou un élément du contexte antérieur auquel ce déterminant renvoie ? En effet, le thème du discours a été mis en place dans la phrase précédente, cf. Le sixiéme [jour], il créa l'homme de bоuё $[\ldots]$, où le pronom personnel il de troisième personne, qui apparaît immédiatement avant l'occurrence, peut être considéré comme le véritable contrôleur, non plus syntaxique mais discursif. Suivant Fournier (1998, p. 286), pour qui « la phrase est en français classique un cadre trop étroit et mal adapté à la description des mécanismes de contrôle référentiel [...] pour l'emploi des modes impersonnels », on aurait donc affaire, dans les cas de cette sorte, à de fausses ruptures partielles, c'est-àdire à des ruptures totales déguisées.

\subsection{Structure phrastique vs organisation discursive}

Selon Marchello-Nizia (1979, p. 46, citée par Seguin, 1993, p. 87) :

Le changement fondamental dans la signification du mot phrase peut être daté des premières années du XVIIIe siècle [...] Le père Buffier écrit une Grammaire françoise sur un plan nouveau $[1709]^{11}$ dans laquelle les acquis de la grammaire générale sont intégrés ; mais ce n'est pas le mot proposition qui apparaît : c'est le mot phrase, ou plus précisément le groupe phrase complète.

Seguin (1993, p. 72) se demande par ailleurs si la « Grammaire [de Régnier-Desmarais, 1705] ne contient pas déjà en germe, par certains côtés, [...] une grammaire de la phrase ». De ce point de vue, la comparaison entre les deux états de langue choisis, français du début du XVIIIe siècle et français contemporain, prend place dans le cadre de «ce que l'on pourrait rapprocher d'un mouvement de grammaticalisation, dans la mesure où la structure phrastique l'emporte peu à peu sur l'organisation discursive » (Combettes, 2000, p. 90).

\section{De certaines ruptures communes au français classique et au français contemporain}

Sont présentées dans cette partie certaines sortes de ruptures qui se rencontrent également en français contemporain. On insistera particulièrement, parmi les infinitifs prépositionnels de temps et de but en rupture (seuls pris en considération dans l'étude), sur la prédominance des seconds sur les premiers. On décrira également brièvement quelques emplois communs de gérondifs en rupture. 


\subsection{Les constructions détachées infinitives en rupture}

\subsubsection{Les infinitifs prépositionnels de temps en rupture}

On relève peu de cas de rupture avec des infinitifs prépositionnels compléments circonstanciels de temps, introduits par avant (que) de ou après. Dagnac (2003) souligne pour le français contemporain ce qui semble également valoir pour le français classique du début du XVIIIe siècle : un comportement différencié des constructions infinitives vis-à-vis de la règle de coréférence, suivant leur valeur circonstancielle, les constructions infinitives compléments de temps apparaissant les plus contraintes. L'infinitif de ces dernières doit notamment être contrôlé par le sujet du prédicat premier, c'est-à-dire le « sujet de surface de la matrice » (Dagnac, 2003, p. 159), ce qui n'est pas respecté par l'occurrence Est-ce que l'on nous accuse de vouloir tout brûler avant que de nous en aller? (Tyssot, p. 39), dans la mesure où le contrôleur, s'il se trouve dans la matrice, n'y exerce pas la fonction sujet, mais la fonction objet direct. De même, l'occurrence Avant que de nous quitter, mon hôte me demanda si je n'avois pas vî la montagne ardente, lorsque je fus aux mines. (Tyssot, p. 194) fait figure d'exception, car, le contrôleur étant à la fois le sujet (mon) hôte et le complément d'attribution me, l' « unicité de l'antécédent » ${ }^{12}$ n'est pas vérifiée. Une autre possibilité, dans ce dernier cas, serait d'attribuer à la construction détachée en rupture, en raison de sa position initiale dans la phrase, un rôle de continuité référentielle, de maintien du thème. On note, d'ailleurs, de façon générale, en rapport à ce contrôle discursif (voir plus bas 4.2), des ruptures d'infinitifs temporels moins rares en français classique qu'en français contemporain (voir RossiGensane, à paraître), mais qui restent toutefois de loin inférieures à celles des infinitifs de but.

\subsubsection{Les infinitifs prépositionnels de but en rupture}

Les infinitifs prépositionnels introduits par pour connaissent les ruptures les plus nombreuses ${ }^{13}$. Selon Dagnac (2003), qui note également cette prépondérance pour le français moderne, les infinitifs prépositionnels compléments de cause en pour et les infinitifs prépositionnels compléments de but, s'ils peuvent être contrôlés par le sujet du prédicat dont ils dépendent, peuvent également l'être par divers arguments internes. Elle suggère (2003, p. 166) que le contrôle de l'infinitif pourrait, dans de tels cas, être de nature sémantique, et non syntaxique. Le contrôle serait effectué, non par une fonction syntaxique (sujet), mais par un rôle sémantique donné. Dans le cas des infinitifs prépositionnels compléments de cause, le contrôleur aurait le rôle sémantique fixe de but ${ }^{14}$. Elle propose (2003, p. 164), pour expliquer la nature sémantique du contrôle, de voir dans les infinitifs compléments de cause des arguments facultatifs du verbe (alors que les infinitifs compléments de temps seraient de vrais circonstants), dans la mesure où le sémantisme du verbe recteur est dans ce cas soumis à des contraintes ${ }^{15}$.

Dans les œuvres examinées, les infinitifs prépositionnels de but en rupture dépendent souvent d'un verbe tel que conduire, envoyer, mener ou mettre, qui renvoie au déplacement d'un être exprimé par le complément d'objet direct, l'infinitif étant contrôlé par ce dernier, cf. Enfin vers les onze heures il envoye un domestique, pour voir doucement s'il dormoit ou non. (Tyssot, p. 172) Si l'on accepte également pour les infinitifs prépositionnels compléments de but l'hypothèse d'un contrôle sémantique, on peut constater que le contrôleur reçoit alors le rôle sémantique de patient (entité animée sur laquelle s'exerce directement le procès, qui est ici plus précisément un déplacement). D'autres rôles sémantiques sont cependant probablement en jeu, suivant la construction du verbe recteur ${ }^{16}$ et la sorte de ruptures. A ce dernier égard, on notera l'unique anacoluthe parmi les infinitifs de but liée à une structure passive : $s i$ elles [nos actions] peuvent devenir bonnes ou mauvaises, ce ne peut être que par raport à de certaines institutions, comme sont celles dont nous venons de parler, et ausquelles elles doivent être mesurées, pour ainsi dire, pour en savoir la juste valeur. (Tyssot, p. 191) Le contrôleur est doté du rôle sémantique d'agent, car il correspond au complément d'agent - non exprimé. La proposition « équivalente » à l'actif est conforme à la règle de coréférence, le complément d'agent étant devenu sujet, par exemple sous la forme du pronom personnel indéfini on, cf. [...] auxquelles on doit les mesurer, pour ainsi dire, pour en savoir la juste valeur. On contrastera le caractère isolé de cette anacoluthe avec les nombreux cas de la même sorte en français contemporain (voir Rossi-Gensane, à paraître), à l'oral mais aussi à l'écrit (et ce 
dans divers genres textuels : journaux, romans (notamment policiers), courriels, articles dans des revues scientifiques...), cf. La bataille doit d'abord être menée maintenant, dans les entreprises, pour conserver le lundi de Pentecôte (Le Figaro, 22 avril 2005), où l'agent pluriel, voire générique, inexprimé, est le contrôleur.

\subsection{Les constructions détachées gérondives en rupture dans quelques emplois}

\subsubsection{Les " gérondifs intellectuels »}

On mentionnera brièvement des cas de « gérondifs intellectuels » (Halmøy, 2003, p. 116), où le participe présent apparent - qui peut être précédé de en -, dénote une activité intellectuelle et a nécessairement un contrôleur - inexprimé - humain, cf. Suposant donc que la terre tourne, les parties les plus agitées doivent être celles qui s'éloignent de son centre avec le plus d'impétuosité [...]. (Tyssot, p. 107) Le gérondif, exophrastique, est alors «le signe d'un regard extérieur porté sur le contenu phrastique » (Guimier, 1996, p. 13).

\subsubsection{Les gérondifs incidents à des noms prédicatifs}

Halmøy (2003) souligne que, contrairement au participe présent qui est toujours incident à un syntagme nominal (remarque qu'il convient cependant de nuancer, puisqu'elle n'est pas valide pour les participes en rupture), le gérondif est, dans l'immense majorité des cas, incident au prédicat verbal ou à la phrase (en tant que complément circonstanciel). Or, on constate que, dans certains cas de rupture, le gérondif est

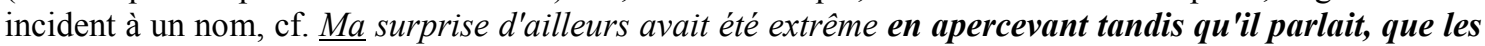
dehors de la caverne ne ressemblaient point à ceux par lesquels j'avais été introduit la première fois. (Prévost, p. 77), où, contrairement à ce qu'exige la règle de coréférence, ce n'est point le sujet ( $m a$ ) surprise, mais le déterminant possessif $m a$, qui contrôle le gérondif. Il s'agit d'incidence à des noms processifs, pas nécessairement déverbaux, mais toujours prédicatifs au sens que leur donne la théorie « lexique-grammaire ». La rupture se produit parce que ces noms ne sont pas accompagnés d'un verbe support, leur sujet étant alors représenté par un génitif subjectif ou par un déterminant possessif. Ainsi, contrastons l'occurrence précédente avec la phrase correspondante, conforme à la règle de coréférence : J'avais eu d'ailleurs une extrême surprise en apercevant...

Remarquons, dans les ruptures de cette sorte - qui se rencontrent également en français contemporain (voir Rossi-Gensane, à paraître), presque exclusivement à l'écrit qui favorise des formes nominales là où l'oral utiliserait des verbes ${ }^{17}$-, la congruence entre prédicat nominal et gérondif, tous deux non temporels. Comme on le verra particulièrement en 4.2 et en 4.3 , la présence de plusieurs propositions favorise les ruptures. Or, le syntagme nominal équivaut ici, au moins sur le plan sémantique, à une proposition, dont le sujet est un déterminant ou un complément du nom, et le prédicat, un nom.

\subsubsection{Les gérondifs repères temporels}

On rencontre quelques cas de rupture concernant les gérondifs repères temporels dont la fonction est «d'indiquer un moment ou un espace de temps qui sert de repère temporel à l'action du verbe fini » (Gettrup, 1977, p. 215). Ces gérondifs, selon Halmøy (2003, p. 118), «jouissent d'une grande liberté syntaxique » et correspondent notamment à des verbes perfectifs de déplacement, cf. Nous nous mîmes en chemin vers le quartier de Mylady Axminster. En allant, je renouvelai la conversation que j'avais eue la veille avec elle, et je priai le vicomte de lui expliquer ce qu'il pensait de sa proposition. (Prévost, p. 104), où aller (utilisé ici sans complément, ce qui n'est plus possible en français moderne) n'est pas contrôlé par le sujet je, mais par nous - qui inclut je, la disjonction étant partielle - dans la phrase précédente. 


\section{De certaines ruptures spécifiques au français classique ${ }^{18}$}

Les ruptures spécifiques au français classique, par rapport au français contemporain, sont liées dans une grande mesure à des thématisations ${ }^{19}$ (voir Rossi-Gensane et Calas, 2006). Or, comme le dit Combettes (2000, p. 91), «c'est la position frontale qui engendre des conflits entre les facteurs textuels et la cohésion syntaxique ». Entre les deux états de langue, le rôle de continuité référentielle, de maintien du thème, des constructions détachées à l'initiale de la phrase ou de la proposition s'affaiblit, selon certaines modalités qui vont être examinées. Seront particulièrement affectées les formes participiales en -ant, dont il va être tout d'abord question.

\subsection{La prédominance des formes participiales en -ant}

En français contemporain, et ce pour divers genres textuels, le taux de rupture des constructions détachées à forme verbale non finie est beaucoup moins important pour les participes, présents ou passés, que pour les gérondifs et les infinitifs prépositionnels. Comme il a déjà été avancé (Rossi-Gensane, 2006), cette différence peut s'expliquer, pour ces deux groupes, par la coïncidence et la non-coïncidence respectives entre point d'incidence et contrôleur. En effet, pour les participes détachés, qui sont des appositions, le nom ou pronom point d'incidence est également le contrôleur ${ }^{20}$. En revanche, pour les gérondifs et pour les infinitifs prépositionnels détachés, qui sont des compléments circonstanciels, le point d'incidence, qu'il s'agisse du prédicat verbal ou de la phrase, et le contrôleur, "normalement » nom ou pronom sujet, diffèrent. Quand le contrôleur et le point d'incidence sont dissociés, la structure concernée subit plus facilement une rupture de construction, puisque la perte du contrôleur ne signifie pas du même coup son non-rattachement à (une unité de) la phrase.

Dans les œuvres examinées du début du XVIIIe siècle, on note un faible nombre de participes passés à forme simple en rupture, qui contraste avec un taux relativement élevé de formes participiales en -antparticipes présents et participes passés à forme composée - dans la même situation. Inversement, ces dernières se trouvent en plus grand nombre que les gérondifs en rupture. Si l'on s'appuie sur la distinction de Combettes (1998, p. 47) entre constructions détachées circonstancielles, qui expriment des relations temporelles ou logiques, et constructions détachées descriptives, ou qualificatives, le nombre encore plus faible de participes passés à forme simple en rupture, par rapport au français contemporain, est en partie imputable au caractère exceptionnel de participes passés de cette sorte à rôle «localisateur ». Or, la plupart des rares participes passés à forme simple en rupture en français contemporain ont une valeur circonstancielle $^{21}$ d'ordre chronologique (voir Rossi-Gensane, 2006, et Rossi-Gensane, à paraître), cf. Arrivés au dessert, Irène demanda : - Alors, ton affaire... ? (Lemaître, P. (2006). Travail soigné. Paris : Editions du Masque, p. 107), où, de façon représentative, le verbe non fini est un verbe perfectif de déplacement, tel qu'on en trouve dans les gérondifs repères temporels (voir plus haut 3.2.3). Cette construction paraît «remplacée » dans les œuvres étudiées de l'époque classique du début du XVIIIe siècle par des formes participiales en -ant composées, cf. [...] je tournai de l'autre côté, et pris la route de Lion. Etant arrivé dans cette fameuse ville, il se rencontra que le marquis De Villeneuve vint souper dans l'hôtellerie où j'étois logé [...]. (Tyssot, p. 222) Autrement dit, l'auxiliaire semble nécessaire à l'expression de la valeur circonstancielle, à la différence de ce qui se produit en français contemporain, qui réalise presque toujours, dans ce contexte de rupture, une économie syntagmatique. On est tenté d'ajouter que tout se passe comme si, dans ce contexte précis, l'auxiliaire était allé au bout de sa grammaticalisation, c'est-à-dire jusqu'à sa propre disparition. Par ailleurs, Halmøy (2003, p. 159-160) souligne, tout au moins pour le français moderne, la possible alternance dans ce contexte, et ce notamment à l'initiale de la phrase, entre participes et gérondifs. Cette alternance est ici exclue, en ne pouvant que difficilement précéder une forme composée (voir plus haut 2.4).

En français contemporain, les rares participes - présents ou passés - en rupture, dans leur immense majorité thématisés, sont presque tous dotés d'une valeur circonstancielle. En effet, dans la mesure où toute apposition sans contrôleur dans la phrase est également privée de point d'incidence - puisque, dans ce cas, contrôleur et point d'incidence coïncident -, cette valeur circonstancielle permet aux participes apposés, en les rapprochant de compléments circonstanciels, d'acquérir un autre point d'incidence, le 
prédicat premier ou la phrase. Dans les œuvres examinées de français classique, presque toutes les appositions en rupture comportent également une valeur circonstancielle. Comme le note Combettes (1998, p. 121), « le système ancien donnait [...] à la construction détachée [en particulier la fonction] de prédication secondaire à valeur circonstancielle. [...] [Cette] valeur sémantique circonstancielle [...] avait pour conséquence des ruptures au niveau syntaxique ». Dans le même temps, "la liaison sémantique circonstancielle, qui s'établit entre la construction détachée et le prédicat qui suit, [allait] jouer un rôle important dans l'incorporation progressive de l'élément périphérique à la structure propositionnelle » (Combettes, 1998, p. 114).

Combettes (2000, p. 102) remarque, pour le moyen français et le français préclassique, qu'un type particulier de construction montre bien «le statut intermédiaire de la [construction détachée], qui n'est pas encore totalement "subordonnée" au prédicat principal, malgré une liaison sémantique forte. Il s'agit des cas où [la construction détachée] suit immédiatement un connecteur », c'est-à-dire un coordonnant ou un subordonnant. C'est précisément dans ces deux contextes qu'apparaissent deux sortes de ruptures en français classique qui ne persistent pas, en tous les cas sous la même forme, en français contemporain (voir aussi Rossi-Gensane et Calas, 2006), et qui vont maintenant être examinées.

\subsection{Les constructions détachées en rupture dans un contexte d'enchaînement de phrases}

On note de nombreuses ruptures de constructions détachées (en premier lieu des formes participiales en ant mais aussi des infinitifs de temps et de but) dans un contexte d'enchaînement de phrases, qui renvoient à des cas traditionnellement dits de coordination de phrases. Le verbe non fini de la construction détachée, au lieu d'être contrôlé par le sujet de la seconde proposition indépendante à l'initiale de laquelle il est placé, l'est par le sujet de la première proposition indépendante, cf. Elle s'était défendue d'abord, en protestant qu'elle ignorait ce qu'il était devenu, mais n'étant point assez ferme pour résister longtemps à de telles instances, il avait tiré d'elle l'aveu de tout ce qui s'était passé. (Prévost, p. 59), ou encore cf. On le fit venir à la cour, et après l'avoir bien examiné, il fut condamné comme le dernier des blasphêmateurs, à aller finir ses jours au fond d'une mine, où il mourut quelque tems après. (Tyssot, p. 155) Dans ce dernier exemple, une lecture moderne pourrait - à tort - mener à voir le contrôleur dans le complément d'agent implicite du verbe au passif qu'est le prédicat premier (et ce malgré le peu de prédilection pour les structures passives, en ce qui concerne les ruptures, des constructions infinitives temporelles, à la différence de ce qui se produit en français contemporain pour les infinitifs de but (voir plus haut 3.1)).

Cette fonction de sujet de la première proposition indépendante est remplie, comme il est fréquent, par le thème, auquel continue de s'appliquer le verbe non fini de la construction détachée. La construction détachée assure donc le maintien du thème. En revanche, en l'absence d'un sujet coréférent pour le prédicat de la seconde proposition indépendante, se produit ensuite un changement de thème, ce qui signifie une rupture référentielle. Or, si, à la suite de Feuillard (1989, p. 214), la phrase est définie comme une «structure constituée d'un prédicat [ou de plusieurs prédicats coordonnés possédant un même sujet] et de l'ensemble des rapports de dépendance contractés autour de ce[s] prédicat[s] [...]», alors, "si l'on se place dans une perspective strictement syntaxique, la coordination entre phrases ne présente pas d'intérêt, dans la mesure où elle ne permet à aucun élément particulier de fonctionner » (Feuillard, 1989, p. 128). Les phrases traditionnellement dénommées coordonnées renvoient à des structures syntaxiquement indépendantes ${ }^{22}$, où la phrase graphique recouvre plusieurs phrases syntaxiques. Lesdits coordonnants, dans ce cas, certes expriment un lien, mais qui, plutôt que syntaxique, est sémantique et textuel. Et, en particulier, marque une continuité entre deux états de choses et ne peut être utilisé que du fait d'une cohésion suffisamment forte.

La « coordination de phrases » n'étant pas reconnue comme pertinente au niveau syntaxique, les ruptures se produisant dans ce contexte d'enchaînement apparaissent comme relevant d'un cas particulier de contrôle discursif. Les ruptures où la construction détachée assure le maintien d'un référent saillant 
subsistent, dans une certaine mesure, en français contemporain (voir Rossi-Gensane, à paraître), notamment à l'oral ou dans des genres textuels écrits « intermédiaires », tel le courriel :

- Nous vous informons que Johnny Halliday viendra au Vinci le mercredi 08/11/2006. Ne pouvant mettre d'option sur son concert, toutes les personnes intéressées sont priées de se faire connaitre. (Courriel, 2005)

mais aussi, exceptionnellement, dans des écrits plus normés, tel le français journalistique :

- Tout le week-end, les pompiers ont continué leur difficile exploration des décombres pour retrouver d'éventuelles victimes. [...] Après avoir étayé une partie effondrée de la cage d'escalier et consolidé les planchers avec l'aide des charpentiers de Paris, le déblaiement des lieux a également pu commencer. (Le Figaro, 18 avril 2005)

On constatera cependant que les ruptures de cette sorte sont beaucoup plus rares qu'en français classique, d'où, entre les deux états de langue, une forte diminution des participes et des infinitifs temporels ${ }^{23}$ non conformes. En outre, ces ruptures n'ont presque jamais lieu, en français contemporain, tout au moins à l'écrit, dans des phrases « coordonnées ». Ce contraste entre, d'une part, français contemporain et, d'autre part, français classique - mais aussi français préclassique et moyen français (voir Combettes, 2000) -, est lié au degré d'intégration de la construction détachée à la structure phrastique. En effet, selon Combettes (2000, p. 102), «dans une séquence : P1 + connecteur + apposition + P2, [...] lorsque l'apposition est intégrée à la structure phrastique, [...] P1 [est lié] à P2 par l'intermédiaire du connecteur, le groupe détaché étant en quelque sorte entre parenthèses et n'intervenant pas dans le rapport logique ». En revanche, lorsque l'apposition est peu ou pas intégrée à la structure phrastique, elle ne peut plus être supprimée parce que «la portée du connecteur s'exerce en fait sur l'ensemble constitué par l'apposition et par P2, et non seulement sur P2» (Combettes, 2000, p. 102). Cette remarque vaut aussi, tout au moins en français classique, pour les compléments circonstanciels. Ainsi, dans Je vous écris ; mais pour vous punir, vous n'aurez de moi que la suite de l'histoire que je n'achevai point hier. (Crébillon, p. 503), le rapport d'opposition ne s'établit pas seulement entre les deux prédicats premiers, la construction détachée étant « indispensable au maintien de la cohérence logique »(Combettes, 1998, p. 113), cf. ?Je vous écris ; mais vous n'aurez de moi que la suite de l'histoire...

On pourrait aussi, pour rendre compte du contraste entre le français contemporain et les états de langue plus anciens, avancer l'hypothèse, en relation avec l'évolution du rôle de la construction détachée, qu'un maintien du thème, une continuité référentielle, entre la proposition précédente et la construction détachée n'est désormais plus perçu comme suffisant pour assurer la cohésion entre deux propositions. Cette liaison forte de la construction détachée avec le contexte de gauche dans le cadre de propositions dites traditionnellement coordonnées est cohérente avec la conception, au début du XVIIIe siècle, de Buffier $(1709, \S 76$, p. 47-48, cité par Seguin, 1993, p. 101), qui distingue, par rapport à la période, la «période imparfaite », qu'il illustre par la vertu est toujours aimable, cependant elle n'est pas toujours aimée, où « une phrase dépend de la précédente, sans que la précédente dépende de la suivante » ${ }^{24}$.

\subsection{Les constructions détachées en rupture dans un contexte d'enchâssement de phrases}

Les ruptures dans un contexte d'enchâssement de phrases affectent presque exclusivement des formes participiales en -ant - seulement éventuellement quelques gérondifs, derrière d'apparents participes présents - et correspondent à un cas particulier de contrôle discursif dans le cadre de la phrase (phrase véritablement syntaxique, et non simplement graphique). Le participe présent « devrait » être contrôlé par le sujet de la proposition subordonnée, à l'initiale de laquelle il est placé, mais il est contrôlé par un terme de la proposition principale, souvent - mais pas nécessairement - le sujet, cf. [...] les autres [routes] ne faisaient qu'augmenter ma peine, parce que, se partageant en diverses branches, j'étais à tout moment dans la nécessité de tenir un nouveau conseil pour délibérer sur celle qu'il fallait suivre. (Prévost, p. 75)

Le participe présent apparaît comme produit de manière différée, dans la subordonnée et non dans la principale, le subordonnant ne semblant pas marquer une frontière. Le contrôleur ne saurait être le point 
d'incidence du participe présent dans la mesure où ce dernier n'entretient pas de relation syntaxique directe avec lui ou avec un autre terme de la proposition principale ${ }^{25}$. Le participe présent est doté d'une valeur circonstancielle (voir plus haut 4.1), ici causale, qui lui fournit un lien avec le contexte de droite : comme elles se partageaient en diverses branches, j'étais à tout moment... Le déplacement du participe présent à gauche dans la phrase, à côté de son contrôleur, permet, conformément à la règle de coréférence, la nette coïncidence de ce dernier avec son point d'incidence, cf. les autres [routes], se partageant en diverses branches, ne faisaient qu'augmenter ma peine, parce que j'étais à tout moment dans la nécessité de tenir un nouveau conseil pour délibérer sur celle qu'il fallait suivre. La phrase obtenue n'est cependant pas strictement équivalente : l'apparente incidence à la proposition du participe présent est supprimée, ce qui peut altérer la coloration circonstancielle de ce dernier, voire mener à une valeur descriptive (glosée par «les autres [routes] qui se partageaient en diverses branches... »).

La proposition subordonnée, comme dans l'exemple précédent, peut être une subordonnée conjonctive circonstancielle ou une subordonnée conjonctive complétive, souvent, mais pas exclusivement, introduite par un verbe de type penser ou de type dire, cf. Songez-vous [...] que rompant avec moi si injustement, [...] je ne voudrois plus voir en vous qu'un homme digne de toute ma haine. (Crébillon, p. 559), ou encore cf. Je fis réflexion que, n'ayant plus rien à attendre du monde, il fallait me former de nouveaux goûts, et chercher ailleurs les plaisirs qu'il me refusait. (Prévost, p. 69)

En français contemporain, les ruptures comparables affectent presque exclusivement des gérondifs (parfois des infinitifs prépositionnels) et se produisent très majoritairement en français parlé. Le prédicat principal est un verbe - parfois un adjectif - de modalité, c'est-à-dire exprimant l'attitude énonciative du sujet parlant vis-à-vis de ce qu'il énonce, cf. J'ai pensé qu'en retouchant son nez et son visage, elle serait plus jolie (oral dans un reportage de journal télévisé, 2005, où le locuteur est un chirurgien). Pour que l'occurrence soit conforme à la règle de coréférence, il faudrait - malgré la présence du subordonnant que - déplacer le gérondif dans la proposition principale, dans laquelle se trouve son contrôleur, qui en est le sujet (comme il a été dit précédemment, la phrase obtenue n'est pas parfaitement équivalente, le gérondif ne dépendant plus de la même proposition et sa valeur circonstancielle étant susceptible d'être modifiée, ici de l'hypothèse à la localisation temporelle). Or, selon Blanche-Benveniste (2004, p. 57), la proposition qui suit un verbe de modalité n'est pas véritablement une subordonnée (que est d'ailleurs éventuellement affaibli) et constitue « la partie centrale de l'énoncé, du point de vue de la syntaxe comme de la signification ». En particulier, le verbe, dit recteur faible, peut apparaître en incise après la " fausse » subordonnée complétive privée du subordonnant que: En retouchant son nez et son visage, elle serait plus jolie, j'ai pensé (ou à l'intérieur de cette «fausse »subordonnée : En retouchant son nez et son visage, j'ai pensé, elle serait plus jolie).

Soulignons que la rupture est, comme il est fréquent, favorisée par la présence, tout au moins apparente, de plusieurs propositions, plus étroitement en rapport pour des raisons syntaxiques ou sémantiques. Si, à la suite de Vion (2004, respectivement p. 103 et p. 106), on distingue nettement entre d'une part modalités, telles que je pense, je crois, il semble..., qui «contribuent directement au sémantisme des énoncés auxquels elles participent », et d'autre part modalisations, telles que les expressions adverbiales probablement, sans doute, certainement..., qui impliquent «un dédoublement énonciatif avec un commentaire réflexif sur l'énoncé », on constate que les ruptures ne s'exercent qu'en relation à des modalités au sens strict. On ajoutera que les verbes de modalité apparaissant dans ce contexte ne sont pas figés, notamment du point de vue du temps, et ne peuvent donc être réanalysés en « marqueurs discursifs propositionnels » (voir en particulier Andersen, 2007). On peut néanmoins suggérer qu'ils s'inscrivent dans un processus de grammaticalisation lié à une métanalyse $\mathrm{e}^{26}$, si l'on admet qu'ils reçoivent alors deux lectures, non parenthétique et parenthétique, associées à une même forme dans la mesure où, en tous les cas en français normatif, l'incise en position initiale n'est pas acceptée, cf. ?J'ai pensé, en retouchant son nez et son visage, elle serait plus jolie. 


\section{Conclusion}

Presque toutes les constructions détachées en rupture en français classique, comme d'ailleurs en français contemporain, sont dotées d'une valeur circonstancielle. Cette dernière autorise notamment à rattacher les appositions thématisées, très nombreuses, au contexte de droite, lesquelles acquièrent ainsi un point d'incidence : le prédicat qui les suit, voire le reste de l'énoncé organisé autour de ce prédicat. En conséquence, en français classique, bien que les constructions détachées thématisées aient un rôle très important de continuité référentielle, de maintien thématique, dont bénéficient au même titre - bien que ce ne soit pas à la même hauteur -, participes apposés et gérondifs et infinitifs circonstants, la phrase a (déjà) une réelle existence, si l'on prend soin de dissocier nettement contrôle et incidence.

Si les anacoluthes persistent dans l'usage contemporain, elles ne sont pas toujours de la même sorte que dans l'usage classique. Le resserrement du cadre phrastique, dont l'affaiblissement du rôle de continuité référentielle de la construction détachée est un aspect, mène à une occupation différente de la position frontale (par exemple par des constructions détachées descriptives (voir Combettes, 1998, p. 121)). Cette évolution a des répercussions sur les anacoluthes dépendantes de la position frontale, désormais plus contraintes, comme nous avons tâché de le montrer, mais n'empêche nullement, en français moderne et contemporain, le développement d'autres sortes de ruptures, telles par exemple celles (brièvement mentionnées) liées au passif, susceptibles d'apparaitre en position autre qu'initiale.

Il serait à présent souhaitable de préciser et de quantifier les tendances dégagées en s'appuyant, notamment pour le XVIIIe siècle, sur un très vaste corpus comportant des genres (roman " sérieux », récit de voyage...) ou sous-genres (par exemple, roman par lettres) textuels variés.

\section{Références bibliographiques}

Andersen, H. L. (2007). Marqueurs discursifs propositionnels. Langue française, 154, 13-28.

Béguelin, M.-J. (éd.) (2000). De la phrase aux énoncés : grammaire scolaire et descriptions linguistiques. Bruxelles : De Boeck Duculot.

Béguelin, M.-J. (à paraître). De l'aveu d'ignorance à l'indéfini: quels impacts pour une théorie de la grammaticalisation? In Rossari, C. (éd.), Actes du colloque Perspectives contrastives et phénomènes de grammaticalisation (2 et 3 octobre 2006), Université de Fribourg.

Blanche-Benveniste, C. (1997). Approches de la langue parlée en français. Gap-Paris : Ophrys.

Blanche-Benveniste, C. (2004). Aménagements progressifs de la syntaxe. In Intercompréhension et inférences, Actes du colloque international EuroSem (2003). Reims : Presses universitaires de Reims, 41-75.

Charaudeau, P. (1992). Grammaire du sens et de l'expression. Paris : Hachette.

Charolles, M., Combettes, B. (1999). Contribution pour une histoire récente de l'analyse du discours. Langue française, 121, 76-116.

Charolles, M., Lamiroy, B. (2002). Syntaxe phrastique et transphrastique : du but au résultat. In Andersen, H. L., Nølke, H. (éds), Actes du colloque international d'Aarhus Macro-syntaxe et macro-sémantique (17-19 mai 2001). Berne : Peter Lang, 383-419.

Combettes, B. (1998). Les constructions détachées en français. Gap-Paris : Ophrys.

Combettes, B. (2000). L'apposition comme unité textuelle et constituant phrastique : approche diachronique. Langue française, 125, 90-105.

Combettes, B. (2003). L'évolution de la forme en -ant : aspects syntaxiques et textuels. Langages, 149, 6-24.

Dagnac, A. (2003). L'interprétation de PRO dans les infinitives circonstancielles : quelques pistes de recherches. In Merle, J.-M. (éd.), Le sujet. Gap-Paris : Ophrys, 159-172.

Feuillard, C. (1989). La syntaxe fonctionnelle dans le cadre des théories linguistiques contemporaines. Thèse d'Etat, Université Paris-V.

Fournier, N. (1998). Grammaire du français classique. Paris : Belin. 
Gettrup, H. (1977). Le gérondif, le participe présent et la notion de repère temporel. Revue romane, XII(2), 210-271.

Grevisse, M., Goosse, A. (1988). Le bon usage. Gembloux : Duculot.

Guimier, C. (1996). Les adverbes du français - Le cas des adverbes en -ment. Gap-Paris : Ophrys.

Guimier, C. (éd.) (1999). La thématisation dans les langues, Actes du colloque de Caen (9-11 octobre 1997). Berne : Peter Lang.

Halmøy, O. (2003). Le gérondif en français. Gap-Paris : Ophrys.

Lecointe, J. (1997). Le style en -ant au XVIe siècle en France: conscience syntaxique et options stylistiques. L'Information grammaticale, 75, 10-14.

Le Goffic, P. (1993). Grammaire de la phrase française. Paris : Hachette Supérieur.

Marchello-Nizia, C. (1979). La notion de «phrase » dans la grammaire. Langue française, 41, 35-48.

Marchello-Nizia, C. (1999). Le français en diachronie : douze siècles d'évolution. Gap-Paris : Ophrys.

Marchello-Nizia, C. (2006). Grammaticalisation et changement linguistique. Bruxelles : De Boeck Duculot.

Prévost, S. (2003). La grammaticalisation : unidirectionnalité et statut. Le français moderne, LXXI(2), 144-166.

Riegel, M., Pellat, J.-C., Rioul, R. (1994). Grammaire méthodique du français. Paris: Presses universitaires de France.

Rossi-Gensane, N. (2006). A propos des constructions détachées en rupture comportant une forme verbale non finie, et plus particulièrement des participes. In Blanvillain, O., Guimier, C. (éds), Les formes non finies du verbe, Tome 1, Actes du $19^{e}$ colloque du Cercle Linguistique du Centre et de l'Ouest (3-4 juin 2005, Caen), Travaux Linguistiques du Cerlico, 19, 131-146.

Rossi-Gensane, N., Calas, F. (2006). Les participes présents détachés en rupture dans Cleveland d'Antoine Prévost d'Exiles. L'Information grammaticale, 111, 30-34.

Rossi-Gensane, N. (à paraître). Register Variation in the Nonstandard Use of Nonfinite Forms. In Beeching, K. (éd.), Sociolinguistic Variation in Contemporary French. Amsterdam : John Benjamins.

Seguin, J.-P. (1993). L'invention de la phrase au XVIIIe siècle. Louvain-Paris : Peeters.

Vion, R. (2004). Modalités, modalisations et discours représentés. Langages, 156, 96-110.

\footnotetext{
* Je remercie vivement les relecteurs anonymes de leurs précieuses remarques et de leurs suggestions très constructives.

${ }^{1}$ Pour les états de langue anciens (moyen français, français préclassique), Combettes (2000, p. 103) considère que les appositions participiales sont « fort proches des faits de subordination».

${ }^{2}$ Soulignons en outre que «le XVIIIe siècle, malgré les injonctions des académiciens, montre une ponctuation très "libre", c'est-à-dire anarchique » (Drillon, J. (1991). Traité de la ponctuation française. Paris : Gallimard, p. 35). Il n'est que de comparer de ce point de vue, par exemple, pour Cleveland, de Prévost, l'édition de J. Sgard et P. Stewart (2003, Paris : Desjonquères) et celle proposée par Frantext pour les gérondifs et les infinitifs prépositionnels. Ainsi, dans l'occurrence suivante: je laissai James dans ma maison, pour les visiter de temps en temps et leur porter les provisions nécessaires à la vie (p. 62), on remarquera que les infinitifs prépositionnels ne sont précédés d'une virgule que dans l'édition récente (alors que, à l'inverse, la virgule qui apparaissait avant le coordonnant est supprimée). Les différences relevées sont notamment liées au choix d'édition opéré par Frantext (ici, pour Cleveland, celle de 1961, Paris : INALF, qui est elle-même une reproduction de celle de 1777, Londres : P. Vaillant). On évoquera par ailleurs les aléas de la numérisation, pouvant parfois donner lieu à des coquilles.

${ }^{3}$ Dans un sens strict, au sein de la grammaire générative, la notion de contrôleur présuppose l'existence d'une

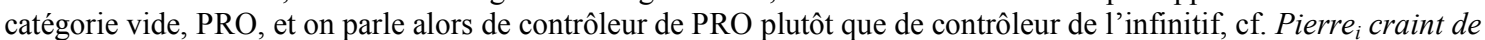
$P R O_{i}$ parler, où la catégorie vide PRO, sujet de l'infinitif, est contrôlée par le sujet Pierre du verbe fini, qui détermine son interprétation. En effet, dans ce cadre théorique, toute phrase grammaticale doit être conforme aux exigences du critère thématique qui établit une relation bijective entre argument et rôle sémantique. La phrase Pierre craint de parler étant grammaticale, il découle du critère thématique que le sujet du verbe parler est présent dans cette phrase, mais aussi distinct de Pierre, déjà sujet de craint qui lui a assigné un rôle d'expérimenteur. Parler ne
} 
peut donc avoir qu'un sujet inaudible, appelé PRO, auquel il assigne un rôle d'agent (voir par exemple Riegel et al., 1994, p. 125-126, pour une définition des principaux rôles sémantiques).

${ }^{4}$ En particulier dans Bouhours, D. (père -) (1674). Doutes sur la langue françoise. Paris : Sébastien Mabre-Cramoisy (réédition Genève : Slatkine reprints, 1972).

${ }^{5}$ En particulier dans Régnier-Desmarais, F.-S. (abbé -) (1705). Traité de la grammaire françoise. Paris : JeanBaptiste Coignard.

${ }^{6}$ En particulier dans Académie Française (1705). Observations sur les Remarques de M. de Vaugelas. La Haye : L'Honoré et Johnson, et Académie Française (1719-1720). Remarques sur le Quinte-Curce de Vaugelas (édition critique par Ayres-Bennett, W., et Caron, P., Paris : Presses de l'Ecole normale supérieure, 1997). Voir aussi AyresBennett, W. (1998). Cela n'est pas construit: l'Académie Française et Vaugelas devant les constructions participiales. In Baudry, J., Caron, P. (éds), Problèmes de cohésion syntaxique de 1540 à 1720. Limoges : Presses universitaires de Limoges, 157-189.

${ }^{7}$ Il s'agit principalement de Cleveland, d'Antoine Prévost d'Exiles (dorénavant Prévost) - texte daté de 1731 d'après Frantext, de Lettres de la marquise de $M^{* * *}$ au comte de $R^{* * *}$, de Claude-Prosper Jolyot de Crébillon (fils) (dorénavant Crébillon) - texte daté de 1732 d'après Frantext, et de Voyages et avantures de Jaques Massé, de Simon Tyssot de Patot (dorénavant Tyssot) - texte daté de 1710 d'après Frantext. Pour Prévost, les numéros de page renvoient à l'édition de J. Sgard et P. Stewart (2003, Paris : Desjonquères); seuls les livres 1 et 2 ont été pris en considération. Pour Crébillon et Tyssot, les numéros de page renvoient aux documents issus de Frantext; pour Crébillon, ne figure que l'extrait allant de la page 331 à la page 611 du document original.

${ }^{8}$ « [...] de Vaugelas à Régnier-Desmarais, la forme de gérondif donnée comme la plus fréquente passe de $V$-ant à en $V$-ant, mais la forme sans en reste acceptée ; ce n'est qu'au début du XVIIIe siècle, avec notamment les Remarques de l'Académie sur le Quinte-Curce de Vaugelas, 1719-1720, que la préposition en sera exigée. » (Fournier, 1998, p. 292)

${ }^{9}$ Dorénavant, le contrôleur de la forme verbale non finie sera souligné dans les exemples où il apparaît et la construction détachée sera mise en gras.

${ }^{10}$ Au sens de Vendler, Z. (1967). Verbs and Times. Linguistics in Philosophy. Ithaca : Cornell University Press, $97-$ 121.

${ }^{11}$ Buffier, C. (père -) (1709). Grammaire françoise sur un plan nouveau (nouvelle édition Paris : Marc Bordelet, 1731).

${ }^{12}$ La propriété d'«unicité de l'antécédent» caractérise, dans la théorie du liage de la grammaire générative, les « anaphores » par rapport aux « pronoms » (voir Dagnac, 2003, pour une synthèse).

${ }^{13}$ Il est sans doute significatif à cet égard que Fournier (1998, p. 290) cite un extrait de l'Académie Française, Remarques sur le Quinte-Curce de M. de Vaugelas, 1719-1720 (p. 275), où la prescription porte sur un infinitif introduit par pour (et non par avant (que) de ou après), complément de but :

Il plaça sur le devant ceux qui portaient des boucliers, \& les fit mettre à genoux pour être moins exposez aux coups de flèches (Q.-C., VII, 162)

Et les fit mettre à genoux pour estre moins exposez. Faute de construction. Pour estre se rapporte par la force de la construction, à celuy qui fait faire l'action, et par le sens il se rapporte à ceux qu'on fait mettre à genoux et qui doivent estre moins exposés. Il falloit donc dire, Et les fit mettre à genoux afin qu'ils fussent moins exposés.

${ }^{14}$ Le rôle sémantique de but renvoie à une entité vers laquelle est dirigé le procès. Dagnac (2003, p. 166) donne les exemples suivants : Paul a été condamné à perpétuité pour avoir tué trois personnes, On a décerné une médaille à Paul pour avoir sauvé trois personnes, où le contrôleur, Paul, reçoit dans les deux cas le rôle sémantique de but.

15 «Les structures infinitives causales reposent sur une idée de sanction / rétribution et plus le verbe est éloigné de ce schéma, moins il est acceptable dans la matrice, cf. Pierre a été condamné pour avoir dealé vs *Pierre a été consolé pour avoir pleuré. » (Dagnac, 2003, p. 164)

${ }^{16}$ Cette idée ne peut être développée dans le cadre de cet article.

${ }^{17}$ Voir notamment Blanche-Benveniste (1997, p. 61-62). 
${ }^{18}$ Insistons sur le fait qu'il s'agit de spécificités du français classique par rapport au français contemporain, et non par rapport à des états de langue plus anciens, dont ces spécificités, évidemment, peuvent être parfois des survivances (voir par exemple Combettes, 2000 ; Combettes, 2003 ; Lecointe, 1997).

${ }^{19}$ Dès la fin du XIVe siècle, le français devient « une langue XSVO à thème(s) en tête », suite au figement de la séquence SV qui «permet à d'autres éléments de commencer la phrase » (Marchello-Nizia, 1999, p. 48-49). S'il va maintenant être évoqué les constructions détachées en rupture en position X, nous ne préciserons pas davantage la valeur de thème de X, qui fait débat (voir Marchello-Nizia, 1999, p. 57-59).

${ }^{20}$ «En tant que forme adjectivale, le participe est incident syntaxiquement à un support nominal, qui est son sujet sémantique (ou contrôleur). » (Fournier, 1998, p. 304)

${ }^{21}$ Il s'agit, pour les participes détachés, qui sont des appositions et non des compléments circonstanciels, d'une valeur circonstancielle au sens large, autrement dit d'un effet de sens circonstanciel.

${ }^{22}$ Pour un point de vue proche, voir également Béguelin (2000) : «C'est [...] dans la dimension macro-syntaxique ou textuelle que la problématique de la relation séquentielle et non hiérarchique entre phrases prend tout son sens » (p. $110) ;$ «[...] les relations de coordination [entre phrases] relèvent d'un niveau d'analyse spécifique, extérieur au domaine phrastique $[\ldots] »($ p. 128).

23 L'exemple ci-dessus est la seule occurrence de rupture totale pour un infinitif temporel dans un corpus journalistique constitué des éditions du Figaro, de L'Humanité et de Libération entre le 8 et le 23 avril 2005.

${ }^{24}$ La période consiste en revanche en « deux ou plusieurs phrases jointes ensemble», cf. la vertu mérite tellement d'être estimée, que nous l'estimons jusques dans nos énemis, où « de ces deux phrases, l'une ici supose l'autre \& en dépend, pour former un sens complet » (Buffier, 1709, § 75, p. 47-48).

${ }^{25}$ Ces contraintes évoquent des notions très utilisées en grammaire générative, tels le domaine et le contrôle local, mais sur lesquelles nous ne pouvons nous étendre dans le cadre de cet article.

${ }^{26} \mathrm{Au}$ sens de Jespersen et de Blinkenberg, comme le rappelle Béguelin (à paraître), c'est-à-dire que la métanalyse suppose « une même forme analysable de deux façons ». 